\title{
Energy Savings Opportunities and Energy Efficiency Performance Indicators for a Serial Production Line
}

\author{
Michael Brundage, Qing Chang^, Shiyao Wang, Shaw Feng, \\ Guoxian Xiao, and Jorge Arinez \\ APMS 2013 International Conference Advances in Production Management Systems \\ Sustainable Production and Service Supply Chains \\ September 9-13, State College, PA \\ \{Michael.Brundage, Qing. Chang, Shiyao.Wang\}@stonybrook.edu, \\ Shaw.Feng@NIST.gov, \{Guoxian.Xiao, Jorge.Arinez\}@gm.com
}

\begin{abstract}
Modern manufacturing facilities waste various energy savings opportunities (ESO) and lack proper performance indicators to measure energy efficiency on the production line. The ESO is an opportunity window calculated from on-line production data, such as production count, machine downtime records, buffer levels, and machine idle status, allowing certain machines to be turned off for energy savings without negatively affecting throughput. New energy efficiency performance indicators are presented that use real time production data to identify the least energy efficiency machine on the line. The energy savings opportunity strategy utilizes the Energy Efficiency Performance Indicators (EEPI) to take the opportunity window for the least energy efficient machine at opportune times, allowing for improvements to be made to the machine, increasing the overall energy efficiency of the line.
\end{abstract}

Keywords: Energy Savings Opportunities, Energy Efficiency Performance Indicators.

\section{Introduction}

With escalating fuel prices and increasing global competition, manufacturing companies are seeking methods to cut costs in any way possible. There are many opportunities to reduce costs in the energy consumption of the facility. These companies are searching for a way to reduce energy cost without sacrificing quality or affecting the yield of their products. The energy consumption in the industrial sector has almost doubled in the past 60 years and accounts for about one-half of the world's total energy consumption $[1,2]$. In the US alone, the industrial sector spent over $\$ 100$ billion in energy costs [3] and was responsible for approximately $34 \%$ of all energy consumed in 2006. In a typical manufacturing plant, the largest source of energy consumption is the production system

\footnotetext{
* Corresponding author.
} 
where $67 \%$ of the total energy cost is attributed to the production process [4]. Being the center of a manufacturing system, production operation directly impacts energy distribution within the manufacturing environment as a whole. The dynamics of the energy demand, largely determines the total energy cost, since the cost of energy (e.g., electricity) actually varies minute-by-minute depending on demand and peak power.

There are few studies that address factory floor planning while considering energy saving opportunities [5-8]. Previous work into this topic has been severely limited with most work focusing on maintaining the quality of the product and the desired productivity while neglecting the energy saving potential. These methods treat the energy consumption as an additional cost term for an optimization problem or the consumption is analyzed as a result of high level decision making and scheduling. The energy consumption is considered a byproduct of the production system and not a main driver in the decision process on the factory floor or the control scheme of the overall system.

Some existing methods, such as the energy treasure hunt developed at GE [9] focused on developing weekend and daily shutdown plans, and managing the leak tag program. Such program is mainly based on non-operation obvious waste, requires expert knowledge on the part of the inspector, and is a "trial and error" manual procedure. There is still a lack of integrated systematic control methodology to drive overall effective energy savings.

One main obstacle in providing an integrated systematic control scheme is the lack of appropriate performance indicators for the facility. While many companies are able to provide key performance indicators (KPI) for a plant, these indicators do not properly address the problem areas on the floor [10-11]. These indicators normally single out the machine with the most energy usage, however this machine may not necessarily be the key issue in terms of energy inefficiency. This is due to the nonlinearity of the production system, which makes it difficult to quantify the impact of individual downtime incidents on the entire operation. The machine center with the most energy usage may not be the least energy efficient machine because of the effects of downtime effects from other machines.

This paper develops and implements new Energy Efficient Performance Indicators (EEPI) that incorporate energy usage from all facets of the manufacturing floor and the facility, and provide energy saving opportunity in real-time production. The EEPI takes into account random downtime events on the manufacturing floor and will allocate the energy usage into two separate categories based on permanent production loss and the lack of synchronization on the floor. This allows the identification of the process that is the most energy inefficient. In addition, the ESO will be applied to save energy and reduce the peak energy consumption so as to reduce overall cost.

The rest of the paper is structured as follows. In section 2 we present background and assumptions. We discuss the energy efficiency performance indicators in Section 3. Section 4 provides simulation studies of the energy opportunities. We dissect the results and provide conclusions and future work in Section 5 . 


\section{Assumptions and Background}

This papers utilizes continuous flow models as seen in Figure (1) [12-14]. The continuous flow model will treat the quantity of jobs in the buffer as varying continuously from zero to the capacity of the buffer as opposed to integer steps. This is done for ease of analysis. The actual system dynamics are not affected by this assumption regardless if the system is continuous or discrete $[15,16]$. For the serial production line as seen above, we can make the following assumptions:

1. Each station $S_{i}$ has a constant rated speed equal to $\frac{1}{T_{m}}$, where $T_{m}$ is the cycle time of the station. A station will run at its rated speed if it is operational and is neither starved nor blocked.

2. A machine is starved if it is operational and its upstream buffer is empty.

3. A machine is blocked if it is operational and its downstream buffer is full.

4. The first machine, $S_{1}$, is never starved and the last machine, $S_{M}$, is never blocked.

5. Each Buffer $B_{2}, B_{3}, \ldots, B_{M}$ have a finite capacity. $B_{2}, B_{3}, \ldots, B_{M}$ denotes the maximum capacity of the buffer.

6. $S_{M^{*}}=\operatorname{argmin}_{m=1, \ldots M} \frac{1}{T_{m}}$ is unique.

7. $W$ is the actual energy consumption for the production system.

8. The total rated power consumption of the line is $P=P_{1}+P_{2}+, \ldots, P_{M}$.

9. $d_{i}^{*}=$ The opportunity window of machine i [17-19].

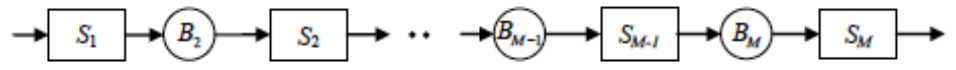

Fig. 1. A Serial Production Line with M Machines and M-1 Buffers

\section{$3 \quad$ Energy Efficiency Performance Indicators}

For the development of the Energy Efficiency Performance Indicators, we must first introduce an energy baseline for the factory. The first step in this process if to define the overall production time of the manufacturing line. The entire production line is dictated by the slowest machine in the system, $S_{m}^{*}=1 / T_{m}^{*}$, where $T_{m}^{*}$ is the cycle time of the slowest machine. If the production count of the line is $M$, then we can define the overall production time as:

$$
t_{p}=\frac{M}{S_{m^{*}}}=M \times T_{m^{*}} .
$$

Knowing that this is the baseline, this means that the actual time it takes to produce $M$ parts will always be:

$$
t_{r} \geq t_{p},
$$

where $t_{r}$ is the actual production time to produce $M$ parts. We can now quantify the energy consumption to a dynamic and static portion in the manufacturing 
process, and categorize the energy consumption in detail (production related and unrelated). The dynamic part is attributed to random disruptions on the line, while the static part is related to synchronization operation. The static part of the energy consumption can be defined as $W_{1}$, which can be seen in equation (3):

$$
W_{1}=W \times \frac{t_{p}}{t_{r}} .
$$

The dynamic portion of the energy consumption due to random downtime events on the line is $W_{2}$ and can be defined as:

$$
W_{2}=W \times \frac{t_{r}-t_{p}}{t_{r}} .
$$

As one can see the total energy consumption $W_{1}+W_{2}=W$. The next step in the process is to distribute the energy consumption at the machine level to aid in developing the Energy Performance Indicator $(E P I)$ for the entire line. The portion of the energy consumption that is due to normal machine operation can be estimated using the power rating of the individual machines. It is defined as $W_{i, 1}$ :

$$
W_{i, 1}=W_{1} \times \frac{P_{i}}{P}
$$

where $P_{i}$ is the power consumption of machine $i$ and $P$ is the rated power consumption of the entire line. Next, we develop the portion of energy that is wasted during permanent production loss, which is $W_{i, 2}$. Permanent production loss occurs when there is a downtime event, $d_{i}$, at machine $i$ that is longer than the opportunity window $d_{i}^{*}$. This will cause the slowest machine to become blocked or starved depending on the location of the down machine. Using this knowledge, the formula for $W_{i, 2}$ becomes:

$$
W_{i, 2}=W_{2} \times \frac{\left(d_{i}-d_{i}^{*}\right)}{\Sigma\left(d_{i}-d_{i}^{*}\right)} .
$$

We can then use $W_{i, 1}$ and $W_{i, 2}$ to find the energy consumption per part at each machine, ECPP:

$$
E C P P=\frac{W_{i, 1}+W_{i, 2}}{M_{i}},
$$

where $M_{i}$ is the production count of machine $i$. If we sum the ECPP for every machine, this will give us the performance indicator for the production line, which we will delineate as the energy performance indicator $\left(E P I_{\text {Actual }}\right)$.

$$
E P I_{\text {Actual }}=\sum_{i=1}^{M} \frac{W_{i, 1}+W_{i, 2}}{M_{i}} .
$$

When there is no energy waste the $W_{i, 2}$ term goes to zero, which gives the energy baseline, defined as EPI Baseline: 


$$
E P I_{\text {Baseline }}=\sum_{i=1}^{M} \frac{W_{i, 1}}{M_{i}} .
$$

As one can see as the production becomes very inefficient and $M_{i}$ becomes small, while $W_{i, 1}$ and $W_{i, 2}$ grow larger the $E P I_{\text {Actual }}$ will grow larger than the baseline. The larger the gap between the baseline and the $E P I_{\text {Actual }}$, and the higher the $E P I_{\text {Actual }}$ the less efficient the production line is performing.

However, the $E P I_{\text {Actual }}$ cannot completely describe the energy efficiency of individual machines. For example, certain machines may have to consume larger energy than other machines, so it cannot be concluded that this machine is energy inefficient. The key is the proportion of the energy consumed in effectively producing products. Therefore, an additional performance indicator is defined as the Energy Efficiency Performance Indicator (EEPI). This performance indicator for an individual machine is equal to:

$$
E E P I_{i}=\frac{W_{i, 1}}{W_{i, 1}+W_{i, 2}} .
$$

\section{Case Studies}

The production system for the case study is a 5 Machine 4 Buffer system (5M4B) with maximum buffer contents of 18 parts for each buffer. The parameters of the line can be seen in Table 1 . The simulation time for this study is 168 hours with an 8 hour warmup time

Table 1. Production Line Parameters

\begin{tabular}{cccccc}
\hline Parameter & $m_{1}$ & $m_{2}$ & $m_{3}$ & $m_{4}$ & $m_{5}$ \\
\hline CT (mins/part) & 3 & 3 & 5 & 3 & 3 \\
MTTR (mins) & 37.5 & 37.5 & 0 & 37.5 & 37.5 \\
MTBF (mins) & 150 & 150 & 150 & 150 & 150 \\
Efficiency (\%) & $80 \%$ & $80 \%$ & $100 \%$ & $80 \%$ & $80 \%$ \\
Power (kW) & 500 & 500 & 100 & 500 & 500 \\
\hline
\end{tabular}

\subsection{Case 1: $d=0$}

The line is first run without any inserted opportunity windows. This will serve as our base scenario without any energy efficiency control strategy. The $E P I_{\text {Actual }}$ for the entire line is calculated using the formula in equation (8) and can be seen in Figure (2).

The solid line indicates the $E P I_{\text {Baseline, which is the energy consumption }}$ without any permanent production loss, calculated from equation (9). The dashed line represents the actual EPI for the entire manufacturing line. This case will allow us to compare the following cases when we insert downtime events into the 



Fig. 2. EPI and EEPI with $d=0$

production line. If the $E P I_{\text {Actual }}$ is greater than this case then the production line is less energy efficient than without any energy opportunity windows. If the gap between the two is smaller than Case 1 then the line is more energy efficient.

To find the least energy efficient machine in the line, we utilize equation (10), and plot the results in Figure (2). This indicates the energy efficiency for individual machines since it takes into account the permanent production loss at each machine due to random downtime events. In this case, machine 2 is the least energy efficient machine as indicated by EEPI since downtime events at machine 2 cause the slowest machine, machine 3 , to become starved, therefore causing permanent production loss. Machine 3 has an EEPI equal to 1.0 because it has no random downtime events, any time not producing parts is due to the other machines causing it to be blocked or starved.

\subsection{Case 2: $d=d_{i}^{*}$}

The next case takes into account inserted opportunity windows. There is permanent production loss for this case because the random downtime events due to machine inefficiencies cause the buffer levels to not reach their full capacity, therefore decreasing the opportunity window. The permanent production loss is $12.3 \%$ when compared to case 1 .

The production count of machine 3 and machine 5 can be seen in Figure (3). Only two machines are shown since each machine except the slowest has the same parameters and would make it difficult to see the inserted opportunity windows in this figure if all were shown at once. Only a portion of the simulation is shown as well to better illustrate the production count of each machine.



Fig. 3. Production Count of Machine $3 \& 5$ for $d=d_{i}^{*}$ 
The inserted opportunity window for machine 5 can be seen from approximately 425 mins - 450 mins where the machine has zero change in production count. The EPI Actual and the EPI Baseline of the entire line with the inserted opportunity windows can be seen in Figure (4).
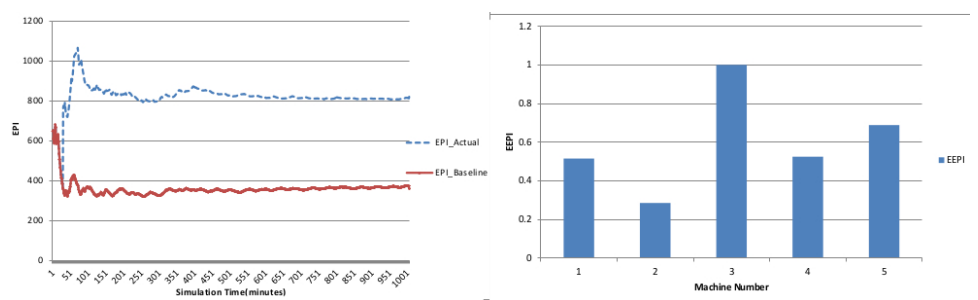

Fig. 4. EPI for the Entire Line with $d=d_{i}^{*}$

As one can see the $E P I_{\text {Actual }}$ and the EPI Baseline decreases for the entire line with the insertion of energy savings opportunities, which is due to the small production loss. The EEPI for each machine can be seen in Figure (4). The $E E P I$ once again illustrates that machine 2 is the least energy efficient. The EEPI cannot be compared to other scenarios, as it is only an indicator of the machine efficiency for each given case. It enables us to identify the least energy efficient machine on the line. Theses results demonstrate that EPI Actual and $E P I_{\text {Baseline }}$ decreases with inserted ESO. This is due to the fact that although the inserted downtime saves the overall energy consumption per part, too much ESO may have the risk to cause more energy consumed by the idling of the bottleneck machine. Therefore, an appropriate ESO strategy without sacrificing production is the key. Furthermore, EEPI captured the portion of the energy used on actually producing parts for each machine rather than downtime and idling, therefore it is used to identify the least energy efficient machine of the production line and help to find the root cause of energy inefficiency.

\section{Conclusions and Future Work}

This paper investigates energy saving opportunities for a serial production line while developing new energy performance indicators for the production line and at the machine level. The performance indicators are tested using simulation studies using three different cases. These studies use different downtime events to prove the concept of the energy opportunity window as well as the energy performance indicators. The indicators are able to correctly identify the machine with the least energy efficiency for each case.

The next step in this research is to develop a control methodology to help alleviate the problem of the least energy efficient machine by utilizing the energy opportunity window or by performing preventative maintenance. 


\section{References}

1. Nidumolu, R., Prahalad, C.K., Rangaswami, M.R.: Why Sustainability is Now the Key Driver of Innovation. Harvard Business Review (September 2009)

2. EIA, 2010, Annual Energy Review 2009 Report No. DOE/EIA-0384 (2009)

3. EIA, US Carbon Dioxide Emissions from Energy Sources 2009 Flash Estimate (2009b)

4. EIA, International Energy Outlook 2009 (2009a)

5. Chen, G., Zhang, L., Arinez, J., Biller, S.: Energy Consumption Reduction in Serial Production Lines via Optimal Startup Schedule. In: 6th Annual ASME International Manufacturing Science and Engineering Conference, Corvallis, OR (2011)

6. Guerrero, C.A., Wang, J., Li, J., Arinez, J., Biller, S., Huang, N., Xiao, G.: Production System Design to Achieve Energy Savings in an Automotive Paint Shop. International Journal of Production Research 49(22), 6679-6785 (2011)

7. Sun, Z., Biller, S., Gu, F., Li, L.: Energy Consumption Reduction for Sustainable Manufacturing Systems Considering Machines with Multiple Power States. In: 6th Annual ASME International Manufacturing Science and Engineering Conference, Corvallis, OR (2011)

8. Fan, K., Uhan, N., Zhao, F., Sutherland, J.W.: A New Approach to Scheduling in Manufacturing for Power Consumption and Carbon Footprint Reduction. In: Proceedings of NAMRI/SME, Corvallis, OR, vol. 39 (2011)

9. Data collection framework on energy consumption in manufacturing. In: IIE Annual Conference and Expo, Orlando, FL

10. Rockwell Software Enterprise Energy Management, Publication RSEEM-PP001AEn-P (November 2009)

11. Optimized energy system management with B. Data, Siemens.com/bdata

12. Sevast'yanov, B.A.: Influence of Storage Bin Capacity on the Average Standstill Time of a Production Line. Theory of Probability and its Applications 7(4), 429438 (1962)

13. Wijngaard, J.: The Effect of Interstage Buffer Storage on Output of Two Unreliable Production Units in Series with Different Production Rates. AIIE Transactions 11(1), 42-47 (1979)

14. Gershwin, S., Schick, I.: Continuous Model of an Unreliable Two-Stage Material Flow System with a Finite Inter-stage Buffer. Technical Report 1039, Massachusetts Institute of Technology, MA (1980)

15. Gershwin, S.: Manufacturing Systems Engineering. Prentice-Hall, Englewood Cliffs (1994)

16. Li, J., Meerkov, S.: Production Systems Engineering, 3rd edn. Wing- Span Press, Livermore (2008)

17. Chang, Q., Xiao, G., Biller, S., Li, L.: Energy Saving Opportunity Analysis of Automotive Serial Production Systems. IEEE Transaction on Automation Science and Engineering (2012), doi:10.1109/TASE.2012.2210874

18. Chang, Q., Biller, S., Xiao, G., Liu, J.: Transient Analysis of Downtimes and Bottleneck Dynamics in Serial Manufacturing Systems. ASME Transaction, Journal of Manufacturing Science and Engineering 132(5), 051015 (2010)

19. Liu, J., Chang, Q., Xiao, G., Biller, S.: The Costs of Downtime Incidents in Serial Multi-Stage Manufacturing Systems. ASME Transaction, Journal of Manufacturing Science and Engineering 134(2), 02101 (2012) 